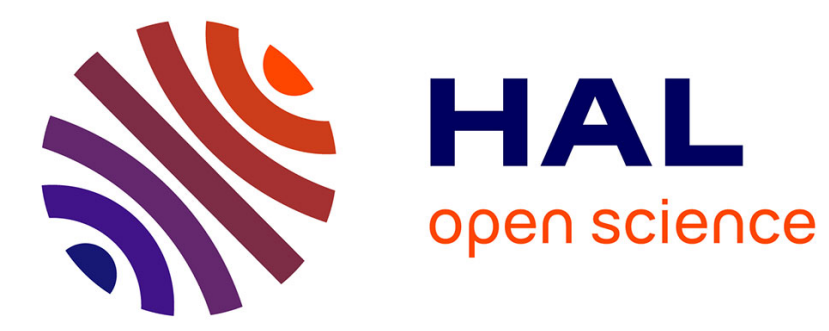

\title{
La langue sans classes de la grammaire scolaire Marie-Anne Paveau
}

\section{To cite this version:}

Marie-Anne Paveau. La langue sans classes de la grammaire scolaire. Le Français Aujourd'hui, 2008, 162, pp.29-40. hal-00582674

\section{HAL Id: hal-00582674 https://hal.science/hal-00582674}

Submitted on 3 Apr 2011

HAL is a multi-disciplinary open access archive for the deposit and dissemination of scientific research documents, whether they are published or not. The documents may come from teaching and research institutions in France or abroad, or from public or private research centers.
L'archive ouverte pluridisciplinaire $\mathbf{H A L}$, est destinée au dépôt et à la diffusion de documents scientifiques de niveau recherche, publiés ou non, émanant des établissements d'enseignement et de recherche français ou étrangers, des laboratoires publics ou privés. 


\title{
La langue sans classes de la grammaire scolaire
}

\author{
Marie-Anne PAVEAU \\ Université de Paris 13 \\ EA 459 CENEL
}

Fainéant se prononce fégnan dans le peuple; mais les personnes cultivées ont droit d'articuler fai-né-ant (P. Martinon, Comment on prononce le français, 1913).

\section{Introduction}

On sait que l'une des spécificités de la France dans son rapport à la langue est le sentiment d'unité : la langue française une et indivisible, comme la Nation, voilà une représentation qui semble résister à toutes les mixités, toutes les multiculturalités et tous les métissages, et, sur le plan proprement linguistique, toutes les variations. Le français standard, ou la représentation qui en est construite, est en effet à la base de tous les enseignements, de la maternelle à l'université, mais aussi de la majeure partie des travaux des chercheurs en linguistique française. Seul ou à peu près, le domaine de la sociolinguistique affronte la difficile question de la variation, difficile sur le plan méthodologique (recueil des données, identification des variantes) et scientifique (comment rendre compte synthétiquement du fonctionnement du système de la langue à partir de données plurielles ?).

Cette représentation unitaire possède évidemment une fonction sociale importante qu'il n'est pas question de remettre en cause : pour des raisons tant pratiques que didactiques, sociales et même idéologiques, c'est bien le français standard qu'il faut enseigner à l'école. Mais cela implique-t-il qu'il ne faut rien dire de la variation, sociale en particulier, et qu'il faut passer sous silence que, dans la langue comme ailleurs, il y a des phénomènes de classe ? qu'il y a un français populaire et un français des classes dominantes ? que les réseaux sociaux ${ }^{1}$, les groupes et les communautés, qui croisent les classes de manière complexe sans les effacer, se définissent aussi par leurs manières de parler, fortement marquées par des déterminismes sociaux?

C'est cette question de la variation sociale au sens le plus classiste ${ }^{2}$ du terme que je voudrais aborder ici, en comparant les grammaires scolaires reposant sur des savoirs linguistiques dits « savants » et les savoirs de la grammaire spontanées la folk grammar, comme disent les Anglo-Saxons, relevant de cette folk linguistics très développée aux États-Unis ${ }^{3}$ et tout juste balbutiante en France ${ }^{4}$, qui intègre pleinement le paramètre classiste.

\section{Où sont passées les classes sociales ?}

On ne rappellera jamais assez que les catégories adoptées par les linguistiques empiriques et sociales ne sont pas immanentes mais directement informées par l'histoire des sciences et des idéologies. C'est particulièrement vrai pour celle de classe sociale, quasiment effacée du discours des sciences sociales,

\footnotetext{
${ }^{1}$ Milroy 1987, Gadet 2007.

2 J'emploie le terme classiste au sens de « social avec stratification en classes ».

${ }^{3}$ Brekle 1989, Preston, Niedzielski 2000.

${ }^{4}$ Paveau 2005, 2007, 2008, Paveau, Rosier 2008, Achard-Bayle, Paveau 2008 (à par.).
} 
comme le montre R. Pfefferkorn dans un ouvrage récent qui reprend la notion marxiste de rapport social (2007).

\subsection{Des classes sans classe}

En sciences sociales, et par conséquent en sociolinguistique, le terme de classe a été remplacé par ceux de position et de réseau. Dans La variation sociale en français ( $2^{\mathrm{e}}$ édition 2007), F. Gadet évite le terme, parlant de «locuteurs favorisés » et « défavorisés », mentionnant « le haut et le bas de l'échelle sociale », et usant du terme position sociale. Elle y signale que la classe (ouvrière, moyenne, supérieure) comme outil d'analyse est trop formelle et réductrice et explique que la sociolinguistique exploite plutôt la notion de réseau en donnant l'exemple du «réseau ouvrier» tel qu'il est par exemple analysé par L. Milroy (1987). Les sociolinguistes parlent volontiers de variation diastratique ${ }^{5}$, terme technique qui efface la dimension idéologique voire politique de classe. Mais en même temps, le terme de classe sociale persiste, et on trouve par exemple classe ouvrière, classe moyenne et classe supérieure dans la présentation de l'usage du ne de négation chez F. Gadet (2007:98). Donc classe ou réseau ? Comme le note R. Pfefferkorn, la disparition de la notion a peut-être plus été un escamotage idéologique qu'une véritable nécessité scientifique, et son retour dans les travaux des jeunes sociologues actuels sur les inégalités sociales qui proposent un questionnement nouveau de l'appareil théorique marxiste aurait tendance à la prouver ${ }^{6}$.

Quoi qu'il en soit, que ce soit sous la forme de la classe, du réseau, de la communauté ou du groupe de pairs, la stratification sociale de la langue n'apparait guère dans la grammaire scolaire, ce qui constitue à la fois une évidence installée tellement nous sommes habitués à l'unité légitim(é)e du standard, et un incroyable écart entre la langue apprise et la langue parlée, qu'elle soit celle des élèves ou de leurs enseignants ${ }^{7}$.

\section{2. « Nantis et pauvres du langage »}

Que l'école ne doive pas coller à la vie et que la langue de l'école ne soit pas celle de l'expérience ordinaire, c'est un principe anciennement acquis et il n'est pas question de le remettre en cause : non, je ne fais pas cours en français parlé ordinaire à mes étudiants, et mes collègues du primaire ou du secondaire non plus. Oui, les manipulations langagières et les apprentissages lexicaux et syntaxiques accomplis à l'école n'ont pas vocation à « servir » directement dans la vie quotidienne, mais à construire une base de savoir langagier avec lequel l'élève pourra jouer, au sens technique du terme, dans sa pratique de la langue. L'amusante séance sur l'imparfait du subjonctif pusse dans le film de L. Cantet Entre les murs (palme d'or à Cannes en 2008) le dit très bien : mais qu'est-ce que vous voulez que j'en fasse de votre pusse, dit en substance une élève, vous me voyez dire à ma mère «que je pusse »? Non, évidemment, pusse servira à autre chose, par exemple à s'approprier le système de la langue.

Mais il me semble qu'il faut faire quelque chose de cette question éminemment sociale (classiste) : chez moi, dit l'élève, non seulement on ne dit pas pusse mais on n'en a jamais entendu parler, c'est en dehors de mon expérience. Elle est décomplexée, mais ce n'est pas le cas des enquêtés de P. Marconot, habitants de la ZUP nord de Nimes :

\footnotetext{
${ }^{5}$ La typologie habituelle de la variation en sociolinguistique comprend cinq critères : diachronique (selon les époques), diastratique (selon les classes ou réseaux sociaux), diaphasique (selon les situations d'interlocution, c'est là que se situent les niveaux de langue), diatopique (selon les aires géographiques, les dialectes par exemple) et diamésique (selon le choix du canal oral ou écrit).

${ }^{6}$ On a beaucoup parlé classe en linguistique jusque dans les années 1980, par exemple chez A. Rey (1972), B. Gardin et J.-B. Marcellesi (1974), F. François (1983), et chez P. Bourdieu bien sûr, dont l'article « Vous avez dit populaire ? » de 1983 publié récemment dans Langage et pouvoir symbolique est fréquemment cité dans les travaux contemporains (2001). Pour une analyse de détail sur cette question et une comparaison avec l'emploi des notions de class, social dialect ou class dialect dans la sociolinguistique américaine, voir Paveau 2008.

${ }^{7}$ Les travaux et réflexions sur l'enseignement-apprentissage de la langue ne mentionnent la plupart du temps que la langue parlée par les élèves et je me suis longtemps demandé quelle était celle de leurs enseignants, qui, contrairement à ce que pensent les auteurs des manuels et des programmes, ne parlent pas tous comme Voltaire, ni même comme Duras.
} 
- ben on parle tous mal de toute façon [...] le vrai français ; y en a plus qui le parlent hein

-c'est pas une langue très recherchée [...] disons que les gens sont gamins - issus de milieu ouvrier ou autre bon ben - ça

- l'ouvrier par lui-même il a il a pas à - parler correctement quoi suffit qu'i se fasse comprendre

- des gens de milieu très très populaire [...] qui parlent le français comme - comment dirais-je ? - bon comme comme parlent les prolétaires hein quelquefois un langage très peu châtié $[. .$.$] des expressions crues [...] un langage une petit peu rude (P.$ Marconot, 1990 : 69).

Il y a donc, comme le dit A. Bentolila dans son lexique un peu spectaculaire, servant une argumentation qui n'est pas la mienne ici, des « pauvres et des nantis du langage »:

Les «nantis du langage »s'encanaillent linguistiquement sans risque et avec la meilleure conscience du monde. Mais ceux dont le vocabulaire est limité et imprécis, ont-ils un réel pouvoir linguistique ? Non, ce sont les «pauvres du langage» condamnés à ne communiquer que dans l'immédiat et dans la proximité (A. Bentolila, 2001, rapport sur l'illettrisme, en ligne).

Je montrerai plus loin, sans trop de mal tellement la chose est visible à l'œil nu, que ces données socialesclassistes n'apparaissent pas dans la grammaire scolaire, ni, d'ailleurs, la plupart du temps, dans la grammaire universitaire, sauf option variationniste déclarée.

\section{La dimension sociale dans les corpus scolaire et folk}

La situation sur laquelle je propose de réfléchir est donc la suivante :

- dans les classes se trouvent mélées (dans un idéal de mixité sociale) ou rassemblées (dans la réalité des ZEP et des établissements de «quartiers difficiles ») des compétences langagières socialement inégales, où se côtoient (ou pas) riches et pauvres du langage, bourgeois et prolétaires du lexique, princes et ouvriers de l'orthographe.

- dans la grammaire scolaire et les programmes qui la déterminent, seule la norme standard est présente reposant sur une langue «déclassifiée », « désocialisée », comme l'indique l'observation de grammaires contemporaines du primaire et du collège, ou contenu des nouveaux programmes de $2008^{8}$. Que les programmes intègrent la dimension énonciative ou proposent une grammaire phrastique plus traditionnelle, l'absence de la variation sociale est en effet la même.

Sans remettre en cause, encore une fois, la fonctionnalité de l'enseignement du standard, il me semble important de comprendre les enjeux et les conséquences de l'enseignement d'une langue parfois peu reliées aux pratiques courantes des élèves, et de réfléchir à l'intégration de la dimension sociale de la langue dans l'enseignement. Cette réflexion est menée depuis longtemps par la folk linguistique, qui peut fournir aux enseignants des cadres pour construire des pratiques pédagogiques intégrant la variation.

\subsection{La folk linguistique : corpus et données ${ }^{9}$}

La folk linguistique est une linguistique pratiquée par des non-linguistes, c'est-à-dire des locuteurs qui n'adoptent pas de regard scientifique sur la langue ${ }^{10}$. Les analyses folk sont constituées de descriptions linguistiques profanes (sur le phénomène de la désignation et le rapport entre la langue et le monde, la hiérarchie entre écrit et oral, la conformité aux règles de la langue, etc.), de prescriptions comportementales souvent normatives voire puristes (dites vs ne dites pas) et d'interventions sur la langue par l'intermédiaire d'usages fautifs ou néologiques qui deviennent des normes car ils proposent des solutions à des difficultés langagières (par exemple les verbes en -tionner). Parmi ces pratiques, la classification sociale est très présente : typologie des mots «vulgaires » ou "élégants », interdictions lexicales ou syntaxiques condamnant le «populaire», relevés ironiques de tournures «snobs» ou de prononciations ouvertes épinglant les classes dominantes.

\footnotetext{
${ }^{8}$ Le corpus d'étude est présenté en fin d'article. Les grammaires sont mentionnées sous leur sigle dans le texte.

${ }^{9}$ La traduction du terme folk posant des problèmes de polysémie (l'expression linguistique populaire est ambigüe), j’ai intégré le terme anglais au français, et je parle désormais de folk linguistique ou de folk grammaire (Paveau 2008).

${ }^{10}$ Pour une définition détaillée voir Brekle 1989, notre article dans le $\mathrm{n}^{\circ} 151$ du Français aujourd'hui (Paveau 2005) et pour de nombreux exemples à tous les niveaux de l'analyse linguistique, voir Paveau, Rosier 2008.
} 
Dès 1983, dans un article désormais très célèbre et fréquemment cité, «Vous avez dit populaire ? », P. Bourdieu réclamait une «science rigoureuse de la sociolinguistique spontanée » (P. Bourdieu 2001 : 137), qui faisait d'ailleurs écho à la «stylistique spontanée » qu'il mentionnait dans Ce que parler veut dire en 1981. Cette sociolinguistique spontanée, c'est cette folk linguistique dans ses dimensions sociales, qui se développe dans de multiples lieux de discours, les conversations orales spontanées bien sûr, mais aussi les multiples blogues et forums sur la langue française, les guides de savoir-vivre, les ouvrages normatifs et puristes sur le bon français, ceux relevant de «l'esprit français », l'ensemble constituant une véritable histoire des mœurs langagières françaises.

Le point commun des éléments de ce corpus est de proposer des commentaires métalinguistiques mettant en œuvre la subjectivité des locuteurs. Trois catégories de données subjectives sont concernés : les intuitions, les perceptions et les sentiments linguistiques. Ces catégories d'observation permettent aux non-linguistes de construire des observables parmi lesquels j'ai choisi d'examiner ici les normes sociales de la langue (correct $v s$ incorrect), les marqueurs sociaux du langage (les catégories «populaire », «bourgeois », «snob», etc., concernant les «classes» mais aussi les réseaux sociaux (jeunes, communautés professionnelles, géographiques, socio-géographiques) et enfin les voix, décrites socialement par les accents, les timbres, et les types d'articulation. La méthode est comparative : je regarde ce que le corpus scolaire et le corpus folk disent de ces trois phénomènes langagiers.

\subsection{La norme sociale : l'évidence du français standard}

Toute la complexité et l'intérêt de la question de la norme langagière est sa duplicité, c'est-à-dire l'ensemble de relations parfois très complexes qui existent entre la norme sociale et la norme linguistique. Dans les grammaires scolaires consultées, aucun problème de ce type : la question de la norme n'est jamais posée et le standard est pro(im)posé sans interrogation, sans réflexion sur la faute et même sans exercices à partir de fautes. «L'enseignement de la grammaire a pour finalité de favoriser la compréhension des textes lus et entendus, d'améliorer l'expression en vue d'en garantir la justesse, la correction syntaxique et orthographique », nous disent les nouveaux programmes de primaire pour le CE2 et le CM (p. 14). Oui, mais de quelle correction s'agit-il ? La variation sociale n'est pas mentionnée et l'argot par exemple, qui pourrait fournir des ressources intéressantes pour la manipulation langagière, est absent : «L'école s'interroge peu sur la signification que le standard revêt pour l'enfant, en prenant pour acquis qu'il souhaite l'acquérir, sans dire en quoi il est désirable » (F. Gadet $2007: 105)^{11}$.

Cet aspect désirable du standard et du correct, on le trouve en revanche abondamment décrit dans le corpus folk : parler correctement est désirable parce que c'est parler comme les dominants, c'est maitriser les codes sociaux et accéder au pouvoir ou se faire une place dans la société. Tous les guides de savoirvivre et les ouvrages sur le bon français contiennent ce discours, fût-il prescriptif et parfois sévèrement conservateur (Le bon français de M. Druon paru en 1999 en est un bon exemple). Le standard y est motivé. On le voit par exemple à ces commentaires sur l'excès d'accents circonflexe trouvés sur le site http://langue-fr.net (graphies conservées) :

Châpeaux bâs !!!

C'est ce qu'on appelle une « faute d'hypercorrection » : à trop vouloir bien faire, on ajoute des accents circonflexes là où il n'en faut pas.

TF1 aime le circonflexe

- Le noble AmphigouriX (21 Prairial an CCVIII, 9 juin 2000 d.c.-d.c.g.) -- Vu chez Pernaut-13h [NDÉ : journal télévisé de la première chaine nationale française], une jeune fille préparant l'option «basque » au bac ; son nom en sous-titre, suivi de future bâchelière. Schtroumpf disait : «Vous voyez que les gens aiment le circonflexe, ils en mettent partout. »

- Maximilien (9-6-2000) -- C'est pour faire plus sérieux. Et ça marche, ça fait plus sérieux. Enfin à première vue, puisqu'effectivement, ça fait plus négligé qu'autre chose.

- Schtroumpfix (9-6-2000) -- Bachotage et rabâchage sont les deux mammelles de la future bachelière.

\footnotetext{
${ }^{11}$ La place me manque pour développer ce point mais comment aborder la multiplicité des textes littéraires français, souvent parfaitement canoniques, qui mettent en scène des parlers populaires ou socialement marqués, sans introduire, à un moment ou à un autre des apprentissages linguistiques, l'idée d'un marquage social de la langue ?
} 
- Clément-Noël Douady (10-6-2000) -- On prévoyait qu'elle allait rater son bac, et entrer dans une usine pour y fabriquer des bâches (nouvelle mode : bérêt basque en toile de bâche).

Mais ce qui est intéressant, c'est que le non-standard, vernaculaire ou argot, l'est aussi : la tradition puriste, toujours ambigüe, fait une place à la «truculence » de l'argot, au plaisir du juron, à la jouissance du parler vulgaire (Paveau, Rosier 2008, chapitre 8). Il s'agit là d'une vision de la langue moins scientifique évidemment mais bien plus riche à mon sens que celle des grammaires scolaires.

Le risque immédiat de ce constat est d'alimenter des enseignements subjectifs, esthétiques, et non "scientifiques », et d'aller dans le sens des propositions gouvernementales de ces dernières années qui réorientent les apprentissages linguistiques vers une normativité traditionnelle plus appréciative qu'objective (rapport sur la grammaire 2006, programme de primaire 2008, programme du collège en consultation 2008). Encore que le terme de traditionnel soit à nuancer : la vieille Souché et Grunenwald de mon enfance, pour parler le dialecte orsennien, proposait une rubrique d'exercice irrégulière intitulée «Le bon usage ». On y trouvait par exemple un exercice sous la forme «ne dites pas vs dites » à propos de l'emploi de l'auxiliaire avoir ou être (1961: 61). Or cette question est proprement sociale et historique, comme le montre bien $\mathrm{C}$. Blanche-Benveniste dans une mise au point intitulée « J'ai descendu dans mon jardin », où l'on apprend que Littré admettait parfaitement «j'ai resté un an à Grenoble », accréditant ainsi le français parlé dit fautif des ouvriers de l'usine Opel (Blanche-Benveniste 2003 : 319). La norme a donc changé et le social a influencé la syntaxe elle-même, si souvent présentée comme une structure intangible. Excellente occasion, à mon sens, d'intégrer le diastratique et le diachronique à l'enseignement-apprentissage du français, avec toutes les adaptations nécessaires au niveau des élèves.

L'honnêteté scientifique oblige en effet à tenir des positions nuancées et difficiles : la question importante n'est pas tant celle de la richesse de l'approche que celle de l'enseignabilité : le marquage social de la langue s'enseigne-t-il avec rigueur ? Je le pense. Empêche-t-il l'apprentissage fondamental du système de la langue ? Je ne le pense pas. Donc oui à l'enrichissement du répertoire social des élèves, et non à l'abandon des critères rationnels pour l'enseignement de la langue, seuls garants de son enseignabilité.

\subsection{Les « niveaux de langage » : une catégorie mal définie}

Si l'on ne trouve aucune donnée diastratique dans nos grammaires, en revanche le niveau diaphasique est systématiquement présent, sous la forme d'un encadré ou d'une page intitulée "Les niveaux de langage », ces derniers n'étant pas vraiment définis mais présentés comme liés aux situations d'interaction.

Dans la NGC, niveau $6^{\mathrm{e}}$, sont présentés trois niveaux assortis d'exemples : familier $\rightarrow>$ boulot, courant $\rightarrow$ travail, soutenu $\rightarrow$ labeur (p. 314). La présentation est suivie d'un développement proscriptif: «Comment éviter le langage familier ? », où l'on apprend que le niveau familier s'emploie seulement à l'oral (retour de la vieille confusion entre oral et populaire) et où plusieurs conseils sont donnés pour soigner son vocabulaire (p. 314 et ss.) :

- en évitant le verlan, l'argot, les abréviations

T'es ouf! T'as vu le fric qu'il a ? -> Tu es fou! Es-tu conscient de sa fortune?

- en évitant les suppressions de syllabes, les répétitions

Il en a un chouette scoot', lui -> Il possède un beau scooter

- en utilisant nous plutôt que on

On a fait une belle balade -> Nous avons fait une belle promenade

Attention!

N'emploie pas nous et on dans la même phrase : Nous, on ne bougera pas -> Nous nous ne bougerons pas

Un peu plus loin, des conseils assortis d'exemples sont également donnés pour « éviter le relâchement » :

- la négation complète et la ponctuation

J'te raconte pas la bécane -> Je n'ose pas te décrire ce scooter

- l'interrogation

T'y crois, toi ? Est-ce que tu crois ce qu'il raconte?

- la suppression de verbes passe-partout comme faire 
Faut te la refaire? -> Veux-tu que je te réexplique?

On explique enfin à l'élève comment rédiger en langage soutenu : «Le langage soutenu s'emploie à l'écrit et à l'oral dans les textes litt, les discours :

- Le vocabulaire est recherché et les phrases sont complexes : J'ignorais que tu avais fait l'acquisition d'un cyclomoteur - La grammaire est toujours respectée et utilise certains modes verbaux comme le subjonctif : Il eût fallu que je pusse t'en parler

Je ne voudrais pas tomber dans la facilité du commentaire ironique, le lecteur appréciera la qualité des « traductions » d'un niveau à l'autre, mais plutôt poser des questions :

- sur la définition de la situation : quelles sont les situations qui impliquent le passage, par exemple, de bécane à cyclomoteur ? Comment sont-elles identifiées ou appréciées par les élèves ? Le paramètre social au sens classiste en est-il vraiment absent ? La GPLE 5e proposait à peu près la même chose en précisant «Comme la situation l'exige » (titre de la page) et «En accord avec la situation d'énonciation » (titre du paragraphe)

- sur la classification des niveaux : où est passé le niveau argotique, autrement appelé populaire, vulgaire, grossier, etc. ? Les merde et les bordel et les trou du cul seraient-ils sortis des dictionnaires ? Curieusement, c'est dans les programmes du primaire qu'on les retrouve : « Savoir ce qu'est un registre de langue ; lors des échanges oraux, transposer tout énoncé spontané des registres familier, argotique ou bas en énoncé appartenant au français standard » (CM1, p. 29). Évités, mais cependant mentionnés, les mots «bas ».

La linguistique folk n'a pas de ces pudeurs ou de ces irréalismes, comme on voudra. L'argot est fréquemment analysé dans ses corpus, et les commentaires ne manquent pas de subtilité sur les usages. Ainsi P. Daninos dans Snobissimo mentionne-t-il les alternances diaphasiques des mondains :

C'est sans doute au cours de ces parties que le dosage [vocabulaire encanaillé] dont je parlais tout à l'heure - du type C'était divin... y avait là une de ces pépées mon vieux c'est bien simple la naissance de Vénus en mieux... je crois bien que ce salaud de Rubi a eu un ticket avec elle marrant quoi - est le plus savant (P. Daninos 1964 : 18-19).

Et les correcteurs du Monde, appartenant à la brillante tradition des non-linguistes typographes français, présentent régulièrement des analyses de l'argot présidentiel, dont voici un exemple :

Sarkozy, ou la folie des glandeurs

Tel un astre venu de son firmament, entouré de ses satellites ministériels et préfectoraux, le président de la rèpe a fondu matutinalement mardi 22 janvier sur un "grand ensemble" de Sartrouville, non loin de Paris, pour y porter sa bonne parole. Entendu ces quelques mots rapportés par la presse audiovisuelle, lors d'un bref échange avec des "djeunes" présents sur place et tout ébahis : son "plan banlieue" leur donnera l'occasion de travailler et d'avoir une formation, "parce que la vie c'est pas de glander". Nous ne ferons pas de commentaire sur la syntaxe du président, ni sur la pénétration de sa pensée, mais seulement sur "glander", verbe d'une rare élégance devenu décidément un fleuron du vocabulaire gouvernemental.

Le gland est d'abord le fruit du chêne, ne l'oublions pas, et ce mot nous vient du latin : glans, glandis, et de bien plus loin, en fait, les linguistes le rattachant à la racine indo-européenne gwele [...]. Le sens actuel, "attendre en vain" n'est attesté qu'en 1941 (avec une forte connotation sexuelle : comme "branler"), et glandouiller, autre parangon du verbe gouvernemental, daterait de 1938. Nous laisserons de côté glandage, glandule et glanduleux, qui existent, mais n'ont pas encore été intégrés dans le discours élyséen.

En conclusion nous dirons qu'un nouveau droit du peuple est dans le collimateur du pouvoir, le "droit de glander" ! Mais que les djeunes pourront toujours répondre avec superbe au président, en paraphrasant F. Mauriac : "Le vrai glandeur ignore les offenses des petits" (http://correcteurs.blog.lemonde.fr/correcteurs/, 24.01.2008).

Il me semble que la grammaire scolaire pourrait utilement puiser dans le fonds de la linguistique profane pour présenter une vision enrichie de la langue, à travers les marqueurs sociaux notamment.

\subsection{Les voix : une grammaire aphone}


Cette remarque concerne également les voix. J'entends par voix non seulement l'oral, mais tous les phénomènes marqués qui le caractérisent : accents sociaux, timbres et articulations. La sociolinguistique savante prend en compte l'oral et ses marquages sociaux mais s'arrête à des constats phonétiques :

Les fautes de liaison les plus généralement perçues, exprimées dans l'expression familière "liaison mal-t-à propos », sont les erreurs que l'on appelle "pataquès », qui connotent très péjorativement un discours. On les appelle, pour les plus fréquentes, cuirs [j'en suis très $t$ aise] et velours [moi z aussi] (F. Gadet $1989: 78)$.

Dans le corpus consulté, la grammaire scolaire ne prend pas l'oral en compte ce qui veut dire qu'on n'entend pas, réellement ou indirectement, les voix du français dans les manuels, à part celle de l'enseignant et des élèves. Une seule grammaire, DTP $3^{\mathrm{e}}$, propose un CD audio avec le livret du professeur, servant à des exercices proposés régulièrement dans le manuel. Par exemple :

- Écoutez attentivement cette conversation entre deux amies. Lou vient de présenter Julien, un jeune homme séduisant mais vaniteux à sa meilleure amie, Emerance. En utilisant le style indirect et le récit de paroles, rapportez en quelques lignes seulement ce que se disent les deux amies. Votre texte commencera de la manière suivante : Dans ce passage, Lou demande à Emerance si... (2003, p. 108, sur l'insertion de paroles dans un texte)

- Lors d'une première audition, relevez les subordonnées relatives dans les passages que vous alelz entendre. Au cousr d'une seconde audition, en vous aisdant en particulier des pauses, précisez lesquelles sont épithètes et les quelels sont apposées (DTP 3e, 2003, p. 185, sur la relative)

- L'animateur de la station de radio «SOS solitude » que vous allez entendre a parfois une façon un peu brusque de parler à ses interlocuteurs. Formulez de maière atténuée ses conseils ou ses demandes en utilisant le conditionnel ou l'imparfait (DTP 3e, p. 145 , sur les modes et les valeurs modales)

Bien que la question sociale ne soit pas posée, l'occasion est éventuellement donnée aux élèves d'entendre des accents sociaux ou régionaux qui les familiarisent avec la variation. La grammaire scolaire actuellement en projet propose quant à elle une conception plutôt théâtrale de l'oral, attentive à la technique plus qu'au marquage social :

Conscient que l'oral s'enseigne, le professeur met en place des exercices variés et progressifs qui permettent aux élèves de travailler la mise en voix, la gestuelle et l'occupation de l'espace. C'est dans ce cadre que prennent place en particulier la récitation (en liaison avec les textes étudiés), la lecture à haute voix, l'exposé, le compte rendu, les échanges organisés (Programme de collège en consultation, $2008: 7$ ).

Du côté folk, les notations classistes abondent: des voix très «Guermantes » décrites par Proust aux accents populaires mis en scène par R. Fallet, en passant par les apertures excessives de Marie-Chantal, les voix, leurs timbres et leurs articulations sont fréquemment épinglées. Deux exemples :

- L'intonation distinguée elle-même est aujourd'hui de mauvais ton, voire douteuse. Elle n'est plus le fait que de quelques annonceuses de la radio dont les mots semblent saupoudrés de strass [...]. Quant au madhâme, si souvent précédé de mes hommâges au temps où les a ne sortaient des bouches bien faites que chapeautés d'un accent circonflexe, il est devenu l'apanage des demoiselles de compagnie, ou de compagnies aériennes (Daninos 1964 : 16).

- Éviter également les liaisons trop accentuées. Parfois même elles sont supprimées : «Commen(t) allez-vous ? 》 a remplacé «Commen-t-allez-vous ? ». Quelqu'un qui dirait en regardant sa montre : «Je m'en vais, il est troi-z et demie » serait un peu ridicule dans le monde et pédant au-dehors.

Enfin, une articulation claire et un léger bégaiement d'allure très « oxonienne » (ne pas dire « oxfordienne ») est d'usage pour raconter une anecdote ou une histoire de chasse (P. Jullian, $1992: 197)$.

Quand on enseigne dans des lieux socialement mixtes, ou des quartiers dits difficiles, ou encore des zones de forte immigration, comme dans mon université à Villetaneuse, on ne peut qu'entendre un français très marqué par des accents divers, pour lesquelles les catégories précises ne sont d'ailleurs pas toujours adéquates : le fameux accent banlieue, accompagné d'une élocution à la mitraillette, est à la fois social, ethnique et générationnel. Les adultes insérés en milieu professionnel le perdent.

Que la grammaire du français soit confiné à un écrit inoui me semble donc poser le problème du réel de la langue, ici de nature sociale.

\section{Conclusion}


Les documents fournis par la linguistique folk sont inestimables et parfois exclusifs, les « linguistes du dimanche » accomplissant un travail, en particulier sur les parlers de classe, que les professionnels ne font pas toujours (pour une présentation détaillée de ce phénomène voir Paveau, Rosier 2008).

L'approche sociale de la langue en classe me semble nécessaire, pour des raisons linguistiques (le français standard n'est pas le français), mais aussi didactiques (l'enseignement du standard est nécessaire mais pas suffisant) et éthiques (l'école n'a pas à mentir sur le caractère profondément discriminant du pouvoir symbolique du langage). Elle est aussi à double tranchant puisqu'elle est offerte aux idéologisations de l'enseignement du français, reposant sur des appréciations normatives, esthétisantes, voire pseudoreligieuse (la «mission» de la grammaire abondamment décrite dans le rapport de novembre 2006). Comme toujours, seule une formation solide des enseignants, intégrant un discours réflexif sur leur propre variété sans déni ni mépris, permet de tenir une position scientifiquement rigoureuse et pédagogiquement efficace.

\section{Bibliographie}

\section{Textes officiels}

Bulletin officiel $\mathrm{N}^{\circ}$ 0, 20 févr. 2008, Les nouveaux programmes de l'école primaire. Projet soumis à consultation, hors-série.

Éduscol dgesco, avril 2008, Collège, Projet de programme, français.

Rapport de mission sur l'enseignement de la grammaire, 2006, A. Bentolila, E. Orsenna, D. Desmarchelier.

\section{Corpus folk}

Blogue «Langue sauce piquante » des correcteurs du Monde : http://correcteurs.blog.lemonde.fr/correcteurs/

Site http://langue-fr.net/index/A/A.htm

DANINOS P. (1964), Snobissimo, Paris, Hachette.

JULLIAN P. (1992 [1958]), Dictionnaire du snobisme, Paris, Bartillat.

\section{Corpus grammaires scolaires (par niveaux)}

Grammaire CM. Pour parler, lire et écrire, 2004, R. Tomassone, C. Leu-Simon, Delagrave (PPLE)

Grammaire pour les textes 5e, 1997, Bordas, Nouvelle collection plus que parfait (GPT 5e)

Grammaire pour lire et écrire 5e, 1997, D. Stissi et al., Delagrave (GPLE 5e)

Grammaire pour lire et écrire 3e, 1999, D. Stissi et al., Delagrave (GPLE 3e)

Grammaire $3^{e}$. Discours, textes, phrase, 2003, E. Pellet et al, Belin (DTP 3e).

Nouvelle grammaire du collège, $6^{\mathrm{e}}, 5^{\mathrm{e}}, 4^{\mathrm{e}}, 3^{\mathrm{e}}, 2007$, coord. C. Dunoyer, C. Stolz, Magnard (NGC).

Grammaire, Cycle d'observation, 1961, A. Souché, J. Grunenwald, Paris, Nathan

\section{Références scientifiques}

ACHARD-BAYLE, G., PAVEAU, M.-A. (dir.), (2008 à par.), Linguistique populaire ?, Pratiques 139-140, Metz, CRESEF.

BENTOLILA A. (2001), L'illettrisme en France, Rapport, en ligne sur

http://sd.alinea.free.fr/Documents/200105paris/linguistique.htm

BLANCHE-BENVENISTE C., 2003, «La langue parlée », dans Le grand livre de la langue française, Paris, Seuil, p. 317-344.

BOURDIEU P. (2001 [1983]), «Vous avez dit populaire? », dans Langage et pouvoir symbolique, Paris, Éditions du Seuil.

BREKLE H.E. (1989), «La linguistique populaire», dans Auroux S. (dir.), Histoire des idées linguistiques, Bruxelles, Mardaga, tome 1, p. 39-44.

FRANÇOIS F. (1983), «Bien parler? Bien écrire? Qu'est-ce que c'est ?», dans François F. (dir.), J'cause français, non?, Paris, La Découverte-Maspero.

GADET F. (1989), Le français ordinaire, Paris, Armand Colin.

GADET F. (2007 [2003]), La variation sociale en français, Paris, Ophrys.

MARCELLESI J-B., GARDIN B. (1974), Introduction à la sociolinguistique. La linguistique sociale, Paris, Larousse.

MARCONOT J.-M. (1990), «Le français parlé dans un quartier HLM », Langue française, 85, p. 68-81. 
MILROY L. (1987 [1980]), Language and social networks, Oxford, Blackwell.

PAVEAU, M.-A. 2005. "Linguistique populaire et enseignement de la langue : des catégories communes », $L e$ Français aujourd'hui, 151, p. 95-107.

PAVEAU, M.-A. 2007. «Les normes perceptives de la linguistique populaire », Langage et société, 121, p. 93 109.

PAVEAU, M.-A. 2008. Le parler des classes dominantes, objet linguistiquement incorrect ? Dialectologie perceptive et linguistique populaire, Études de linguistique appliquée 150.

PAVEAU M.-A., ROSIER L., 2008, La langue française. Passions et polémiques, Paris, Vuibert.

PFEFFERKORN R. (2007), Inégalités et rapports sociaux. Rapports de classes, rapports de sexes, Paris, La Dispute.

PRESTON D., NIEDZIELSKI N., 200o, Folk Linguistics, Berlin, New York, De Gruyter.

REY A. (1972), « Usages, jugements et prescriptions linguistiques », Langue française 16, p. 4-28. 